\title{
Random Auxetic Porous Materials from Parametric Growth Processes
}

\author{
Jonàs Martínez \\ Université de Lorraine, CNRS, Inria, LORIA \\ 615, rue du Jardin Botanique, 54600 Villers-lès-Nancy, France
}

\begin{abstract}
We introduce a computational approach to optimize random porous materials via parametric growth processes. We focus on the problem of minimizing the Poisson's ratio of a two-phase porous random material, which results in an auxetic material. Initially, we perform a parametric optimization of the growth process. Afterward, the optimized parametric growth process implicitly generates an auxetic random material. Namely, the growth process intrinsically entails the formation of an auxetic material. Our approach enables the computation of large-scale auxetic random materials in commodity computers. We also provide numerical results indicating that the computed auxetic materials have close to isotropic linear elastic behavior and physical tests revealing auxetic behavior.
\end{abstract}

Keywords: Porous media, inverse design, random auxetics, growth processes

\section{Introduction}

Auxetic structures are materials with a negative Poisson's ratio: when stretched, they expand perpendicularly to the applied force 26, 29, a seemingly counterintuitive property. Auxetic materials find applications in multiple fields thanks to their excellent shock absorption, fracture toughness, or vibrational absorption 61, 51, 25, 30, 49, 45, among other reasons. A substantial amount of research is devoted to designing auxetic mechanical materials [25, 12 58 that derive their physical properties from the particular arrangement of their small-scale geometry. Recent manufacturing technologies can fabricate complex small-scale structures and therefore manufacture auxetic materials.

Random materials offer some significant advantages compared to the more widespread periodic materials. In particular, they are more resilient to fabrication-related symmetry-breaking imperfections [44], can smoothly and seamlessly grade material properties [28, are well suited to manufacture isotropic structures [40, 21, are excellent candidates for energy-absorbing applications [10, 39, 23, and allow to compute the material geometry efficiently 34 . While a repeating periodic structure defines most auxetic materials, a distinct line of research is otherwise interested in random auxetic materials 36 since they offer certain advantages over periodic structures [46, 62, 27]. Auxetic polymeric foams 29, 8, were reported in the 80s and are widely used in industrial applications. The most common process to obtain auxetic foams is compressing a conventional flexible cellular foam to force the cell ribs to buckle, producing a reentrant structure heated to its softening temperature [9, 1]. The geometry of cellular foams is usually idealized and modeled with Voronoi diagrams 17, and some works studying auxetic foams start by modeling a compressed Voronoi diagram [32, 15. Alternative methods to produce random auxetic materials consider sheets with random perforations [18] or random fiber networks produced by electrospinning [13].

A recent research direction tackles the minimization of Poisson's ratio of finite two-dimensional random networks consisting of nodes connected by bonds. Reid et al. 48. proposed to prune network bonds iteratively, further improved in [4] by modifying, in addition, the position of the network nodes and the stiffness of the network bonds. Hagh et al. 19 starts from a planar triangulation and iteratively removes bonds while avoiding the creation of reentrant polygons. Liu et al. 33. proposed an iterative change of the bond stiffness. Pashine et al. [43] presented a network aging process that decreases the Poisson's ratio. All these methods assume that the network's elastic behavior is close to isotropic during the iterative optimization process.

We advocate for a different computationally efficient perspective on computing two-dimensional auxetic random materials. We propose to compute a porous material 52 ] with a solid and void phase through a parametric growth process. The growth process is compactly defined by a random point process and two parametric functions controlling the growth law. Our goal is to optimize these two functions' parameters to minimize the Poisson's ratio of the resulting porous material. Importantly, this optimization is only carried out once and allows the immediate generation of random auxetic materials afterward through the process of growth, requiring no further optimization process. This is in opposition to prior methods 44, 47, 19, 33, 43, that proceed with iterative optimization, rendering the computation infeasible for large-scale materials. We enable the computation in commodity computers of large-scale 
random auxetic porous materials with close to isotropic behavior (see Figure 1).

Our research is influenced by 35 that considered a parameterized growth process to obtain periodic porous materials. Notwithstanding, a number of differences distinguish us from 35. First, we consider the case of random porous materials. Second, we formulate a parametric optimization to deal with the particular case of minimizing the Poisson's ratio. Third, we introduce a novel parameterized set that controls the cease of cell growth and considerably improves the results. Finally, we suggest a hypothesis on the link between certain symmetries of the growth process and the emergence of random porous materials close to isotropic elasticity.

\section{Growth process}

We consider a growth process [1] in which nuclei are born at the same time. Each nucleus, a point $x \in \mathbb{R}^{2}$, is the origin of a cell. Cells grow according to a growth law and are forbidden to overlap. At the end of the growth, the union of all cells corresponds to the porous material's void phase, while its complement in $\mathbb{R}^{2}$ corresponds to the solid phase. We start by describing how the nuclei are placed in space and how the law of growth is defined.

A two-dimensional point process $\Phi \subset \mathbb{R}^{2}$ defines the nuclei. We consider two different point processes: a homogeneous Poisson point process with intensity $\lambda>0$, and a hardcore point process in which the points are forbidden to lie closer together than a minimum distance $D>0$. In particular, we consider the random sequential adsorption (RSA) model 60, where disks with radius $\frac{D}{2}$ are iteratively added as long as they do not overlap any previously added disk. The center of the disks corresponds to the point process, and its intensity is $\lambda=\frac{4 A}{\pi D^{2}}$, where $A \approx 0.547$ is the area fraction where saturation occurs [11].

Cells grow through a uniform scaling of a compact set $\mathcal{S} \subset \mathbb{R}^{2}$ centered on each nucleus. The growth ceases whenever and wherever a cell comes into contact with another (see Figure 2). This type of growth process has been well studied in the literature when $\mathcal{S}$ is a convex set and is also known as the Voronoi growth model 42. Our central idea is to allow $\mathcal{S}$ to be non-convex, enabling the growth of porous materials with more diverse shapes.

In addition, we control how close to each other cells can grow, with what we call the maximum Euclidean radius of growth (see Figure 3). Let $B_{r} \subset \mathbb{R}^{2}$ be a closed Euclidean disk with radius $r$ and centered at the origin. Let $x$ be a point in the boundary of a growing cell. The growth ceases at $x$ if $x+B_{r}$ intersects another growing cell.

We start by defining $\mathcal{S}$. Let $\mathcal{S} \subset \mathbb{R}^{2}$ be a compact star-shaped set with respect to the origin $O=(0,0)$. For any point $x \in \mathcal{S}$, the line segment $[x, O]$ is contained in $\mathcal{S}$. The boundary of $\mathcal{S}$ can be parameterized in polar coordinates by a continuous and periodic function $\psi_{\mathcal{S}}:[0,2 \pi] \mapsto$ $\left[r_{\min }, r_{\max }\right]$, where $0<r_{\min } \leq r_{\max }$ are the minimum and the maximum Euclidean distance from $O$ to the boundary of $\mathcal{S}$.

Following [35, we parameterize $\mathcal{S}$ with a few known values $\left(\alpha_{i}, l_{i}\right)_{i>0}$ of the function $\psi_{\mathcal{S}}\left(\alpha_{i}\right)=l_{i}$, and we call them radial spans. For simplicity, we always consider $m>$ 0 equally spaced radial spans separated by an angle of $\beta_{m}=\frac{2 \pi}{m}$ and starting at an angle of zero $\alpha_{1}=0$. Let $\alpha_{j k} \in[0,2 \pi]$ be an angle we seek to interpolate, lying between $\left(\alpha_{j}, l_{j}\right)$ and $\left(\alpha_{k}, l_{k}\right)$, such that $j=\left\lfloor\alpha_{j k} \beta_{m}\right\rfloor$ and $k=(j+1) \bmod m$. The relative position $t$ of $\alpha_{j k}$ between $\alpha_{j}(t=0)$ and $\alpha_{k}(t=1)$ is given by the fractional part $t=\alpha_{j k} \beta_{m}-\left\lfloor\alpha_{j k} \beta_{m}\right\rfloor \in[0,1)$. Then, we interpolate the value of $\psi_{\mathcal{S}}\left(\alpha_{j k}\right)$ with the following cubic Hermite spline on the unit interval

$$
\psi_{\mathcal{S}}\left(\alpha_{j k}\right)=\left(2 t^{3}-3 t^{2}+1\right) l_{j}+\left(-2 t^{3}+3 t^{2}\right) l_{k}
$$

The interpolation ensures that $\psi_{\mathcal{S}}\left(\alpha_{j k}\right)$ lies between $l_{j}$ and $l_{k}$ by setting the starting and ending tangents of the spline to zero. Besides, we can impose rotational symmetries to the interpolation. Figure 4 illustrates the interpolation procedure. The distance induced by $\mathcal{S}$ from a point $p$ to a point $x$ [35] allows us to define the cell growth process compactly

$$
d_{\mathcal{S}}(p, x)=\frac{\|x-p\|}{\psi_{\mathcal{S}}(\angle(x-p))}
$$

where $L(\cdot)$ is the angle of a vector with respect to the horizontal axis, and $\|\cdot\|$ is the Euclidean norm. $d_{\mathcal{S}}(p, x)$ is the factor by which we have to uniformly scale $\mathcal{S}$, centered on $p$, such that $x$ lies on its boundary. We always consider that $p$ is a cell nucleus and $x$ is any point of the cell emanating from $p$.

A star-shaped set $\mathcal{S}^{*}$ parameterizes the maximum Euclidean radius of growth. We consider a polar function $\psi_{\mathcal{S}^{*}}:[0,2 \pi] \mapsto\left[r_{\min }^{*}, r_{\max }^{*}\right]$, where $0<r_{\min }^{*} \leq r_{\max }^{*}$ are the minimum and maximum Euclidean radii of growth. Let $x$ be a point in the boundary of a growing cell emanating from $p \in \Phi$. The growth ceases at $x$ if $x+B_{\psi_{\mathcal{S}^{*}}(L(x-p))}$ intersects any other growing cell. Thus, $\mathcal{S}^{*}$ determines when to cease one cell's growth process as it approaches other cells (see Figure 3).

The minimal distance between two points in a Poisson point process can be arbitrarily small. Thus, a cell can be empty as its growth was forbidden through $\mathcal{S}^{*}$ from the beginning of the growth process. For a hardcore point process, the minimal distance between two points is at least $D$. Thus, it suffices to enforce $D>r_{\max }^{*}$ in order to guarantee that all cells are allowed to grow.

It is challenging to formulate a continuous growth law 3 . since both $\mathcal{S}$ and $\mathcal{S}^{*}$ are not necessarily convex, and $\mathcal{S}^{*}$ imposes an atypical constraint on the growth process. We instead resort to a discrete growth process formulation 2, where the cells grow in discrete steps. Therefore, our growth process produces a discrete random set. The general principle is to assign each cell a disjoint discrete set of points. 

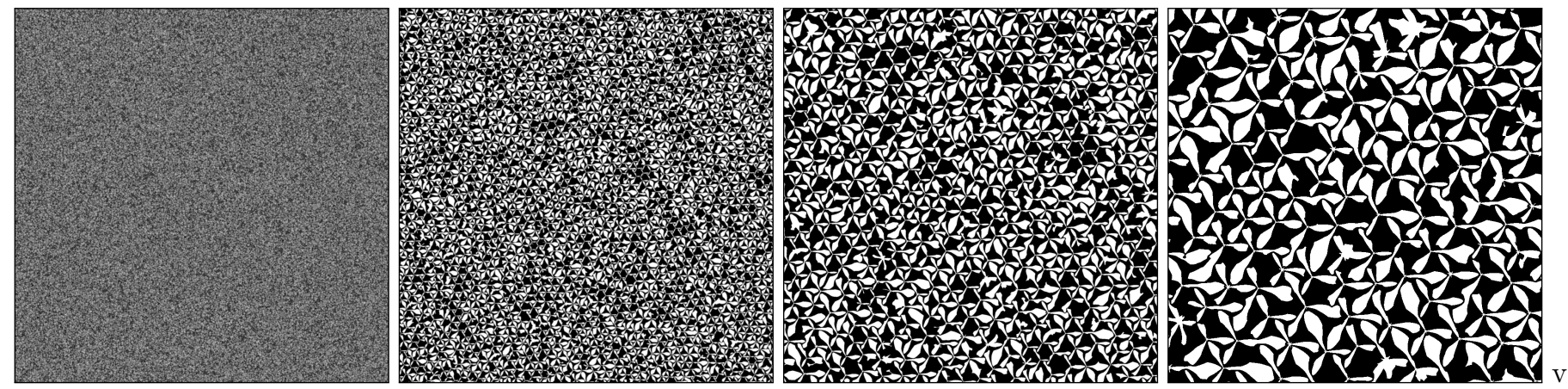

Figure 1: From left to right: large-scale random auxetic porous material with increasing close-up views. The growth process has 43590 nuclei, and the computation took 26 minutes on a laptop using a single CPU core.

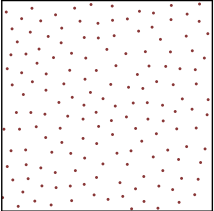

$\Phi$

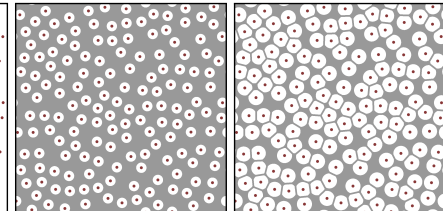

Cell growth

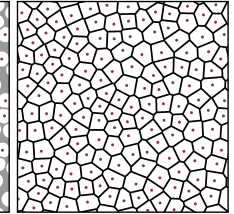

Porous material
Figure 2: In this example, given a point process $\Phi$, the cells grow according to the uniform scaling of a Euclidean disk.

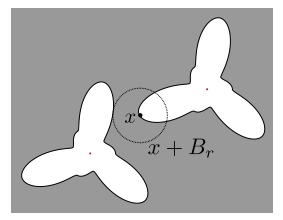

Figure 3: Growth of two cells. The growth ceases at $x$ since the disk $x+B_{r}$ intersects another growing cell.
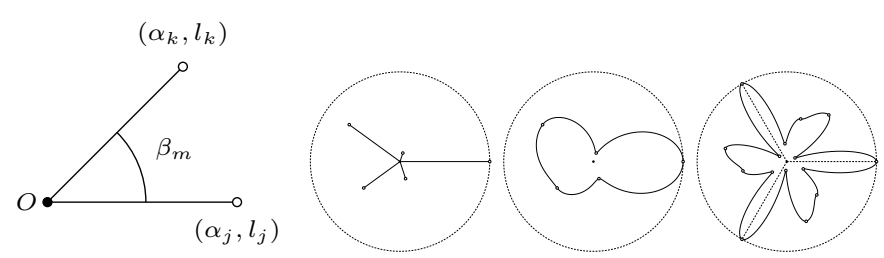

Figure 4: Star-shaped set parameterization and interpolation with five equally spaced radial spans. Leftmost figure: imposing three-fold rotational symmetry.

More precisely, we consider an integer lattice $L_{W}=\mathbb{Z}^{2} \cap$ $W$ where $W$ is a bounded subset of $\mathbb{R}^{2}$. Let $\Phi_{W}=\Phi \cap W=$ $\left(p_{1}, \ldots, p_{g}\right)$ be the subset of nuclei in $W$. The set of lattice points belonging to the cells is given by the union $C_{W}=$ $\bigcup_{i=1}^{g} C_{W}^{i} \subset L_{W}$, where $C_{W}^{i}$ corresponds to the lattice cell points associated with the nuclei $p_{i}$. Lattice points are incrementally added to $C_{W}$ throughout the discrete growth process. Let $S_{1} \subset \mathbb{R}^{2}$ be the unit square centered at the origin and let $\oplus$ denote the Minkowski sum operator.

We consider that the porous material's void phase is given by $C_{W} \oplus S_{1}$, while the solid one is given by $\left(L_{W} \backslash\right.$ $\left.C_{W}\right) \oplus S_{1}$. If $W$ is a rectangle, both phases can be compactly represented with a binary digital image. In all illus- trations, the void phase is colored in white and the solid phase in black. Figures 5 and 6 show some examples of the growth process. The size of the solid phase can be regulated by $\mathcal{S}^{*}$. For instance, lower values of $r_{\min }^{*}$ will lead to narrower porous materials.
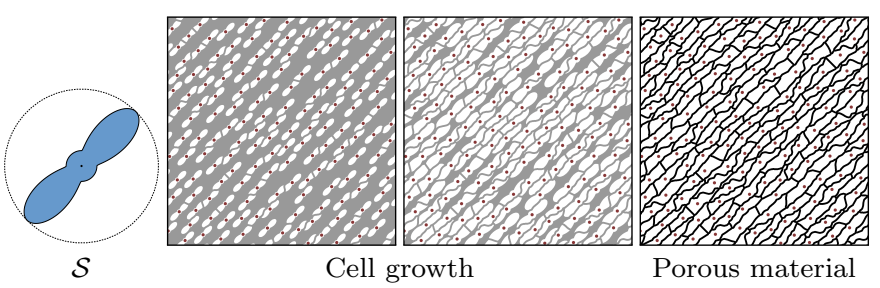

Figure 5: The growth process is given by the point process of Figure 2 and the uniform scaling of $\mathcal{S}$ at the left. In this example, $\mathcal{S}^{*}$ is a Euclidean disk.
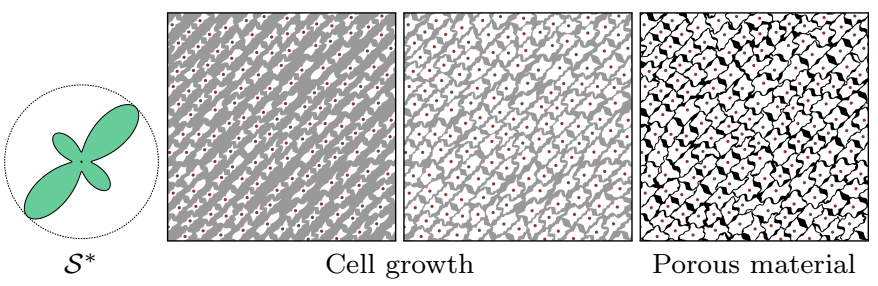

Figure 6: Growth process given by the point process of Figure 2 the uniform scaling of $\mathcal{S}$ in Figure 5 and the maximum Euclidean radius of growth $\mathcal{S}^{*}$ at the left.

In the following, we describe in more detail Algorithm 1 . which defines the process of discrete growth. An ordered set $Q$ contains tuples $\{x, p\}$ where the first element is a lattice point of $x \in L_{W}$ and the second one is a nucleus point of $p \in \Phi_{W}$. $Q$ is ordered according to the distance $d_{\mathcal{S}}(p, x)$. The tuples in $Q$ identify the points where cell growth might take place. The growth process is divided into two parts, the nucleation phase and the growth phase.

Nucleation. Before the growth starts, for all $p_{i} \in \Phi_{W}$, we insert in $Q$ the tuple $\left\{x_{i}, p_{i}\right\}$, where $x_{i} \in L_{W}$ is the closest lattice point to $p_{i}$. Ideally, $L_{W}$ should be sufficiently large, so no two points in $\Phi_{W}$ are closest to the same lattice point in $L_{W}$. 
Growth. The discrete growth process is simulated as follows. Let $\left\{x, p_{i}\right\}$ be the tuple in $Q$ with the smallest distance $d_{\mathcal{S}}\left(p_{i}, x\right)$ among all tuples.

- (Line 7) First, we remove $\left\{x, p_{i}\right\}$ from $Q$.

- (Lines 8-11) Second, if $x$ does not already belong to any cell and the Euclidean disk $B_{\psi_{\mathcal{S}^{*}}\left(\angle\left(x-p_{i}\right)\right)}$ does not contain any other cell point, we add $x$ in $C_{W}^{i}$. That is, the lattice point $x$ now belongs to the cell emanating from $p_{i}$.

- (Lines 12-14) Finally, candidate points of growth around a local neighborhood of $p$ are inserted in $Q$. More precisely, we insert in $Q$ the four tuples $\left\{x_{n}, p_{i}\right\}$, where $x_{n}$ is a lattice point in the 4 -connected neighborhood of $x$ in $L_{W}$.

The previous steps are iteratively done until $Q$ is empty.

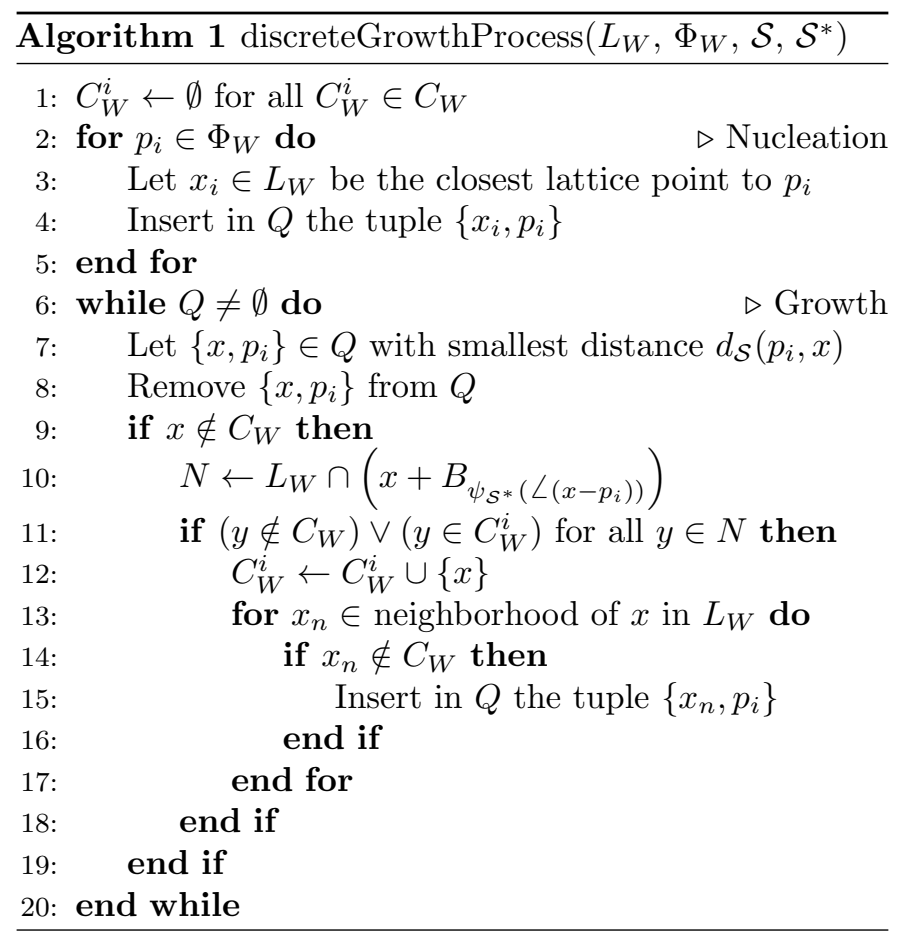

Computational complexity of Algorithm 1. Let $n$ be the number of points of $L_{W}$ and $g$ be the number of nuclei of $\Phi_{W}$. Since we assume that $n>g$, the number of iterations of the nucleation loop (lines 2-5) and the growth loop (lines $6-20)$ is of order $n$. The size of $Q$ is $O(n)$. If $Q$ is implemented as a heap priority queue, then the insertion and removal of elements in $Q$ can be done in logarithmic time (lines 4, 7, and 15). The loop of line 10 (i.e., for all $y \in N)$ has $O(1)$ time complexity since it depends on the constant size of $\mathcal{S}^{*}$. The loop of line 13 also has $O(1)$ time complexity (four iterations). Thus, Algorithm 1 has $O(n \log (n))$ worst-case time complexity. In practice, we observe a quasi-linear runtime with respect to $n$ (see Section 4.3 primarily because the size of the queue $Q$ remains comparatively small in proportion to $n$.
Periodic boundary conditions. Optionally, when $W$ is a rectangle, periodic boundary conditions can be easily imposed by considering that $L_{W}$ is a periodic rectangular lattice as well. If $\Phi$ is an RSA point process, the random adsorption of disks must be performed considering the periodicity. All results shown in this article feature periodic boundary conditions.

Cell regularization. Algorithm 1 is guaranteed to produce cells $C_{W}^{i}$ that are 4 -connected sets. However, we have observed that when $\mathcal{S}^{*}$ is not convex, a cell may have a genus higher than zero (i.e., has "holes"). Holes lead to a solid phase composed of more than one connected component, which is undesirable. To resolve this, we fill any hole that a cell may have. Formally, we consider the complement of the unbounded component of the complement of a cell (see Figure 7). We denominate this last step cell regularization. Numerical results show that cell regularization has, on average, little impact (see Section 4.1).

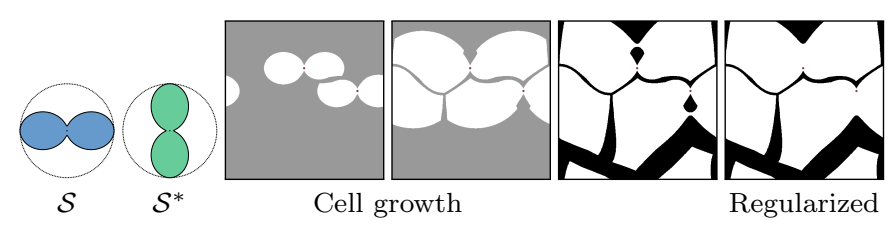

Figure 7: Left: $\mathcal{S}$ and $\mathcal{S}^{*}$ governing the growth process of two nuclei. Middle: evolution of the growth. In this example, the holes arise because of the high anisotropy of $\mathcal{S}$ and $\mathcal{S}^{*}$. Right: cell regularization, the two cell holes are filled.

\section{Elastic behavior of random porous materials}

We study the elastic behavior, assuming linear elasticity and plane stress 55, considering that the stresses in the orthogonal direction to the plane are negligibly small (e.g., a thin plate). We apply a procedure known as stochastic homogenization [6] that seeks to approximate the average elastic behavior of random materials. Hooke's law represents the elastic behavior of materials with the linear relation $\sigma=C \epsilon$, where $\sigma$ is the stress, $\epsilon$ is the strain, and $C$ is the elasticity tensor (a symmetric and positive definite matrix), in Voigt notation 59.

$$
\left[\begin{array}{c}
\sigma_{1} \\
\sigma_{2} \\
\sigma_{12}
\end{array}\right]=\left[\begin{array}{lll}
c_{11} & c_{12} & c_{13} \\
c_{12} & c_{22} & c_{23} \\
c_{13} & c_{23} & c_{33}
\end{array}\right]\left[\begin{array}{c}
\epsilon_{1} \\
\epsilon_{2} \\
2 \epsilon_{12}
\end{array}\right]
$$

Periodic homogenization [4] seeks to find the elasticity tensor characterizing a periodic composite material defined from a periodic cell, as the length of the cell tends to zero. In the periodic case, the homogenization is defined on a finite domain (periodic cell), while in the random case is defined on the whole space $\mathbb{R}^{2}$ and cannot be reduced to a problem posed on a finite domain [7].

We approximate the homogenized elasticity tensor coefficients of a random porous material using the so-called 
"cut-off" techniques. Consider a periodized cut $[0, s]^{2}$ (a square) of a stationary random material and its corresponding homogenized elasticity tensor $C^{s}$. It was shown in [7] that $\lim _{s \rightarrow \infty} C^{s}$ converges, almost surely. Nevertheless, $s$ needs to be bounded for computation. In our discrete setting, the cut size $s$ is a positive integer that corresponds to the pixel resolution $s \times s$ of the porous material image. In all results, we consider a constant $D=80$ for the RSA point process and $\lambda=\frac{4 A}{\pi D^{2}} \approx 0.00011$ for the Poisson point process. Therefore, both point processes have similar intensities.

We compute the homogenized elasticity tensor $C^{s}$ with a publicly available numerical homogenization method based on the finite element method and considering plane stress properties [5]. The input is an image with values identifying each distinct phase of the periodic material. We impose periodic boundary conditions on the growth process to produce a suitable input image. The solid phase is a linear elastic isotropic material with unit Young's modulus $E=1.0$ and Poisson's ratio $v=0.3$.

Let $\mathbb{E}[\cdot]$ be the expectation of a random variable. We estimate the value of $\mathbb{E}\left[C^{s}\right]$ with the average of $k$ independent realizations of the random porous material giving $\left(C^{s}\right)_{i}, 1<i \leq k 6$

$$
\mathbb{E}\left[C^{s}\right] \approx \overline{C^{s}}=\frac{1}{k} \sum_{i=1}^{k}\left(C^{s}\right)_{i}
$$

Let $\operatorname{std}\left(C^{s}\right)$ be the standard deviation of the $k$ independent realizations. A range of plausible values for $\mathbb{E}\left[C^{s}\right]$ is $\left(\overline{C^{s}} \pm 1.96 \frac{s t d\left(C^{s}\right)}{\sqrt{k}}\right)$ for a confidence interval with probability $95 \%$ (see Figure 8). In order to evaluate the accuracy of the stochastic homogenization [24, we examine the statistical fluctuations of the material porosity $p \in[0,1]$ (the area fraction of the void phase) for a given $k$ and $s$. In particular, we consider the coefficient of variation $c_{v}(p)=\frac{s t d(p)}{\bar{p}}$ that indicates the extent of variability of the porosity in relation to the mean. Increasing the value of $k$ and $s$ most likely decreases $c_{v}(p)$. Following [54, we consider that the approximation is sufficiently accurate if $c_{v}(p)$ is below a maximum predefined one. In all the following results, we always consider $k=60$ realizations.

\subsection{Isotropic elasticity}

Since our goal is to minimize the Poisson's ratio of an isotropic material, we give a brief presentation of how we determine if the average tensor $\overline{C^{s}}$ is close to isotropic, and if so, what are its defining parameters $E$ and $v$. An isotropic material has an elasticity tensor $C^{i s o}$ with only two independent components, $c_{11}^{i s o}$ and $c_{12}^{i s o}$

$$
C^{i s o}=\left[\begin{array}{ccc}
c_{11}^{i s o} & c_{12}^{i s o} & 0 \\
c_{12}^{i s o} & c_{11}^{i s o} & 0 \\
0 & 0 & \frac{c_{11}^{i s o}-c_{12}^{i s o}}{2}
\end{array}\right]
$$

For plane elasticity, the relation between the tensor components $c_{11}^{i s o}, c_{12}^{i s o}$ and the Young's moduli $E>0$ and the

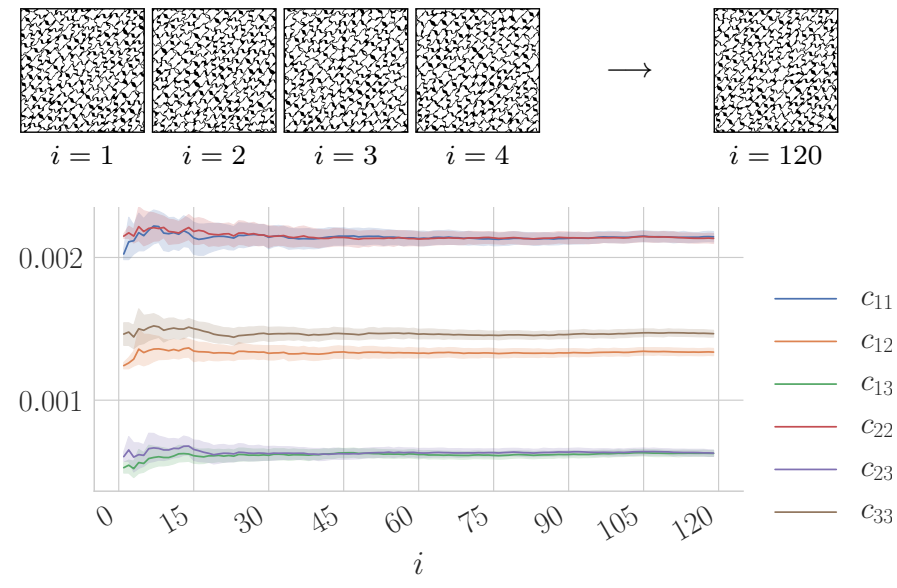

Figure 8: Approximation of the elasticity tensor of the random porous material of Figure 6 in a square with size $s=1200$, and 120 independent realizations. Top: Some realizations. Bottom: mean and confidence interval of each tensor component. As the number of realizations $i$ increases, the mean stabilizes, and the confidence interval becomes narrow. The coefficient of variation of porosity is $c_{v}(p)=0.00409$.

Poisson's ratio $v \in[-1,1]$ is given by

$$
E=\frac{\left(c_{11}^{i s o}\right)^{2}-\left(c_{12}^{i s o}\right)^{2}}{c_{11}^{i s o}} \quad v=\frac{c_{12}^{i s o}}{c_{11}^{i s o}}
$$

In practice, it never occurs that the average tensor $\overline{C^{s}}$ is perfectly isotropic. Thus, we seek to determine its closest isotropic tensor under some norm [37]. We consider the Frobenius norm for a $3 \times 3$ matrix $A$

$$
\|A\|_{F}=\sqrt{\sum_{i=1}^{3} \sum_{j=1}^{3}\left|a_{i j}\right|^{2}}
$$

and minimize the Frobenius norm between $C^{i s o}$ and the average tensor $\overline{C^{s}}$

$$
\min _{\substack{i s o \\ c_{11}^{i s o}, c_{12}^{i s o}}}\left\|\overline{C^{s}}-C^{i s o}\right\|_{F}
$$

We provide in the Appendix A the closed-form expression of $c_{11}^{i s o}, c_{12}^{i s o}$ from $C^{\text {iso }}$ minimizing Equation 8. We also need a measure of the deviation of $\overline{C^{s}}$ from perfect isotropy. We consider the normalized measure $\delta_{i s o}$ used in practice [57, 62

$$
\delta_{i s o}=\frac{\left\|\overline{C^{s}}-C^{i s o}\right\|_{F}}{\left\|\overline{C^{s}}\right\|_{F}} \geq 0
$$

where $\delta_{\text {iso }}=0$ indicates perfect isotropy and increasing values indicate divergence from isotropy.

\section{Results}

\subsection{Three-fold symmetry and isotropy}

It is known that two-dimensional periodic structures with three-fold rotational symmetry (invariant to rotations 
of $\frac{2 \pi}{3}$ ) lead to an isotropic linear elastic behavior [14. We hypothesize that a resembling property holds in our instance of random growth.

A two-dimensional point process $\Phi$ is stationary if $\Phi+x$ has the same probability distribution as $\Phi$ for all $x \in \mathbb{R}^{2}$. $\Phi$ is isotropic if $R(a) \Phi$ has the same probability distribution as $\Phi$, for all rotations $R(a)$ of angle $a \in \mathbb{R}$ around the origin. The Poisson point process and the RSA point process are stationary and isotropic point processes [11, 53].

Let us consider a rotation of $R\left(\frac{2 \pi}{3} n\right)$, for $n \in \mathbb{Z}$. The probability distribution of $\Phi$ is invariant to a rotation $R\left(\frac{2 \pi}{3} n\right)$. If $\mathcal{S}$ and $\mathcal{S}^{*}$ are three-fold symmetric, then $R\left(\frac{2 \pi}{3} n\right) \mathcal{S}=$ $\mathcal{S}$ (same for $\mathcal{S}^{*}$ ). Given a point $p \in \Phi$ then we have

$$
R\left(\frac{2 \pi}{3} n\right)(p+\mathcal{S})=R\left(\frac{2 \pi}{3} n\right) p+R\left(\frac{2 \pi}{3} n\right) \mathcal{S}=R\left(\frac{2 \pi}{3} n\right) p+\mathcal{S}
$$

i.e., the rotation of $\mathcal{S}$ centered at $p$ around the origin keeps $\mathcal{S}$ and $\mathcal{S}^{*}$ invariant. This suggests that the probability distribution of the discrete random set given by the growth process is close to being stationary and invariant to rotations $R\left(\frac{2 \pi}{3} n\right)$, which leads us to the following hypothesis.

Hypothesis 1. Let $\Phi$ be a stationary and isotropic point process. Let $\mathcal{S}$ and $\mathcal{S}^{*}$ be three-fold rotational symmetric. For $s \rightarrow \infty$, the discrete growth process described in this article gives a porous material with $\delta_{\text {iso }}$ close to zero. Namely, the porous material has close to isotropic linear elastic behavior.

We numerically tested Hypothesis 1 for an extensive set of porous materials with different sets of $\mathcal{S}$ and $\mathcal{S}^{*}$, and increasing size $s$ (see Figure 9). There is strong evidence that as $s$ increases, the average value of $\delta_{i s o}$ decreases, and it appears to tend towards a value close to zero.

In all the subsequent results, we consider a square image of side length $s=1200$ pixels. This size gave among all the tests performed in Figure 9 a maximum coefficient of variation of porosity of $c_{v}(p)=0.03519$ for the Poisson point process, and of $c_{v}(p)=0.01118$ for the RSA point process.

We also evaluate the impact of cell regularization over the porosity by measuring the relative number of filled cell holes. Let $\hat{p}$ be the porosity of the porous material before performing the cell regularization. We consider the relative measure $\zeta_{p, \hat{p}}=\left|\frac{\hat{p}-p}{\hat{p}}\right| \geq 0$. For all the results in Figure 9 the maximum value of $\zeta_{p, \hat{p}}$ is 0.06157 and the mean is 0.00433 for the Poisson point process, and maximum 0.01948 and mean 0.00041 for the RSA point process. This indicates that the effect of cell regularization on the porosity is almost negligible, particularly for the RSA point process.

\subsection{Parametric optimization}

By this point, we have all the ingredients to formulate the parametric optimization of the growth process. Let $\overline{C^{s}}\left(\psi_{\mathcal{S}}, \psi_{\mathcal{S}^{*}}\right)$ be the average elasticity tensor of a porous material parameterized by the functions $\psi_{\mathcal{S}}$ (star-shaped distance) and $\psi_{\mathcal{S}^{*}}$ (maximum Euclidean radius of growth). Let $\mathbb{F}: M \mapsto \mathbb{R}$ be the objective function, where $M$ is a $3 \times 3$ symmetric matrix. Our objective is to minimize

$$
\arg \min _{\psi_{\mathcal{S}}, \psi_{\mathcal{S}^{*}}} \mathbb{F}\left(\overline{C^{s}}\left(\psi_{\mathcal{S}}, \psi_{\mathcal{S}^{*}}\right)\right)=\frac{c_{12}^{i s o}}{c_{11}^{i s o}}
$$

where $c_{11}^{i s o}, c_{12}^{i s o}$ are derived from $\overline{C^{s}}\left(\psi_{\mathcal{S}}, \psi_{\mathcal{S}^{*}}\right)$ considering Equation A.4. To attain a physical behavior close to isotropic elasticity, we constrain $\mathcal{S}$ and $\mathcal{S}^{*}$ to be three-fold $=$ rotational symmetric (see Hypothesis 1 .

The input of function $\mathbb{F}$ is expensive to evaluate and has no closed-form. In this case, Bayesian optimization 38. is often a good choice to tackle the optimization. In particular, we have used Bayesian optimization using Gaussian processes as implemented in the Python library scikitoptimize [56]. Both functions $\psi_{\mathcal{S}}$ and $\psi_{\mathcal{S}^{*}}$ are parameterized with $m=8$ radial spans. We set the first radial span of $\psi_{\mathcal{S}}$ to a constant value of 1 , removing an unnecessary degree of freedom. Finally, both $\psi_{\mathcal{S}}$ and $\psi_{\mathcal{S}}$ are concatenated into a single optimization variable to optimize $\psi_{\mathcal{S}}$ and $\psi_{\mathcal{S}}$. simultaneously. We set a maximum number of 150 evaluations of function $\mathbb{F}$ and 50 random initial guesses.

Figures 10 to 12 provide minimization results of the Poisson's ratio with increasingly larger optimization domains of $\psi_{\mathcal{S}^{*}}$ and a constant optimization domain of $\psi_{\mathcal{S}} \in$ $[0.05,1.0]$. We distinguish two clear trends from the optimization results. First, the RSA point process can achieve a lower Poisson's ratio $v$ compared to the Poisson point process. Second, increasing the function $\psi_{\mathcal{S}^{*}}$ domain leads to a lower Poisson's ratio since we expand the space of achievable porous materials. Overall, we recommend using a hardcore point process instead of a Poisson point process to reach a lower Poisson's ratio and lower variance of the material properties using the same area (see Figure 9).

\subsection{Computational efficiency}

We evaluate the efficiency of the growth process computation by performing some timing tests shown in Table 1 . For a range of $n=[1,100]$ million pixels, we observe a nearly linear correlation between $n$ and computing time. Based on the statistics shown in Table 1, we consider that this is mainly explained because the relative average size of the queue $Q$ is below $4 \%$ (column (c)), amortizing the logarithmic cost of insertion and deletion of elements in $Q$. In addition, the relative time spent computing the cell regularization is below 1\% (column (a)), showing that the bulk of the computational cost is in the growth algorithm.

Finally, we observe different scaling laws among the tests, depending on the input sets $\mathcal{S}$ and $\mathcal{S}^{*}$ and the point process type. We infer that these differences are explained mainly by a trade-off between the number of iterations in the main loop (column (b)) and the average number of iterations in line 11 of Algorithm 1 (column (d)). Both statistics are tightly related to $\mathcal{S}^{*}$ : a larger area of $\mathcal{S}^{*}$ implies fewer iterations of (b) at the expense of more iterations of (d). 

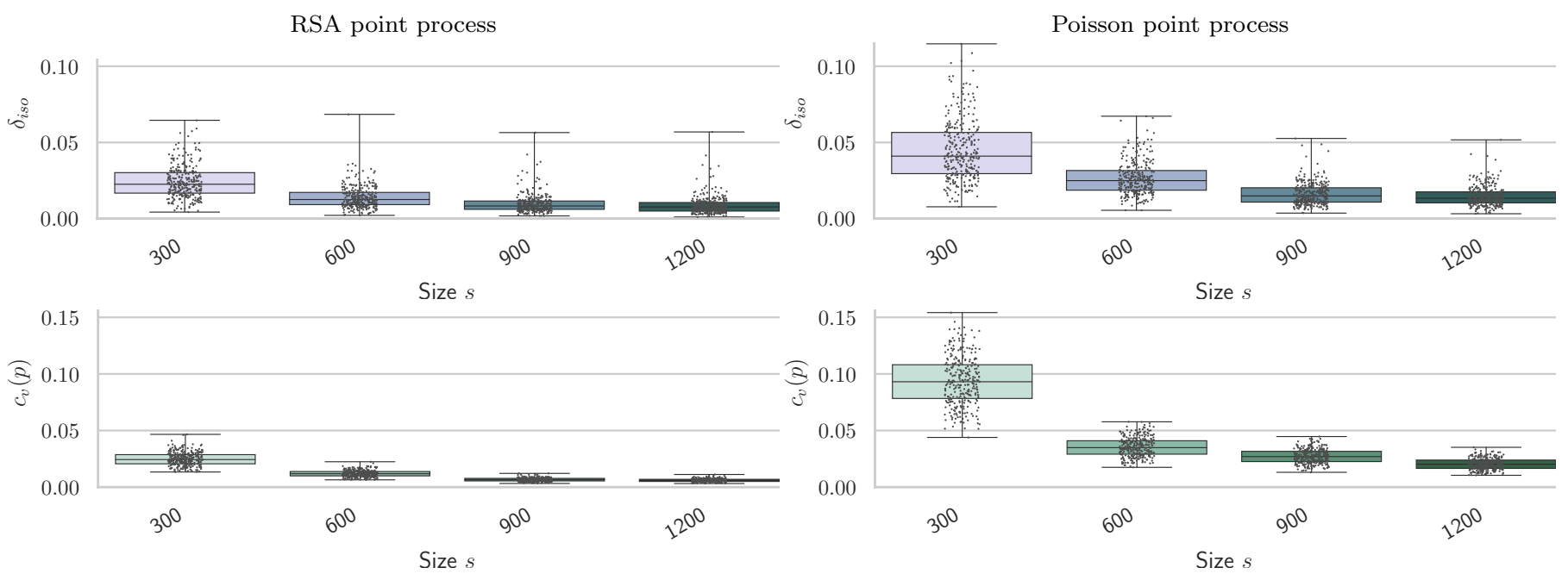

Figure 9: Analysis of the deviation of isotropy $\delta_{i s o}$ and coefficient of variation of the porosity $c_{v}(p)$ for a large number of porous materials and two different point processes (left and right columns). $\mathcal{S}$ and $\mathcal{S}^{*}$ are three-fold rotational symmetric. The colored boxes encompass the interquartile range of values of $\delta_{\text {iso }}$. We consider 4 different sizes $s$, going from 300 to 1200 For each point process and size, we enumerate 300 different random radial spans in the range $\psi_{\mathcal{S}} \in[0.05,1.0]$ and $\psi_{\mathcal{S}^{*}} \in[4.0,40.0]$, and 8 different equally spaced angles in [0, $\left.\frac{2 \pi}{3}\right]$ (three-fold symmetry) considering a total of $2 \cdot 4 \cdot 300=2400$ different porous materials. We observe a clear trend of the average value of $\delta_{i s o}$ and $c_{v}(p)$ getting closer to zero as the size $s$ of the domain increases. We also notice lower average values with the RSA point process compared to the Poisson point process.

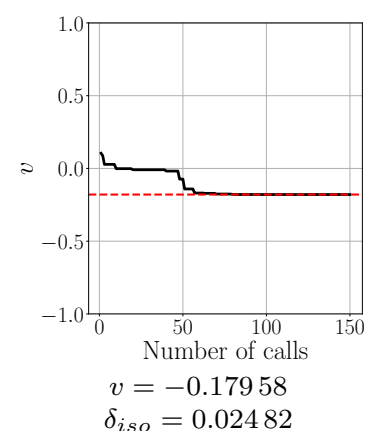

$v=-0.17958$
$\delta_{i s o}=0.02482$

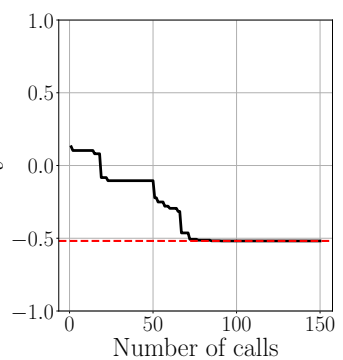

$v=-0.51848$

$\delta_{\text {iso }}=0.04823$

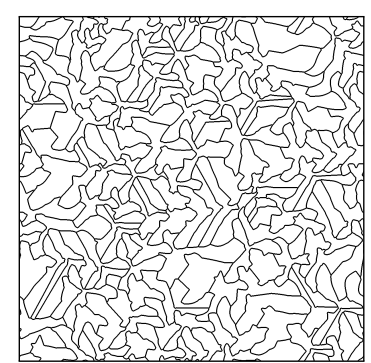

Poisson

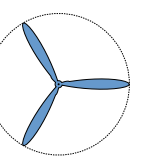

$\mathcal{S}$

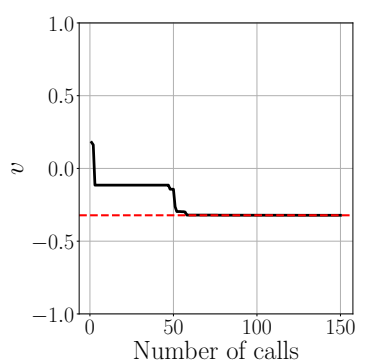

$v=-0.32203$

$\delta_{\text {iso }}=0.03154$

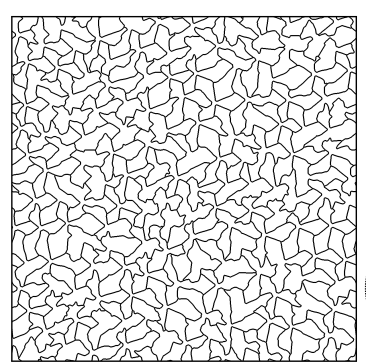

RSA

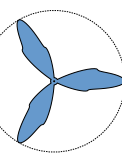

$\mathcal{S}$

Figure 10: Optimization with constant $\psi_{\mathcal{S}} *=4.0$.

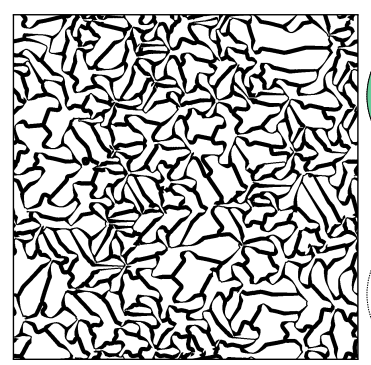

Poisson

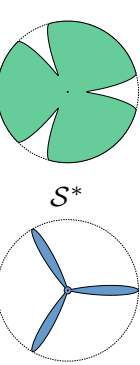

$\mathcal{S}$

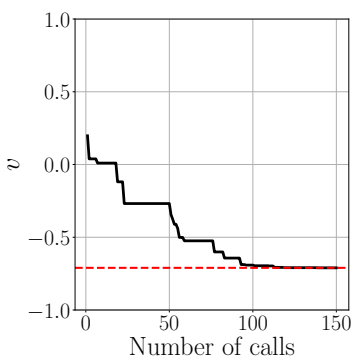

$v=-0.71022$

$\delta_{\text {iso }}=0.01515$

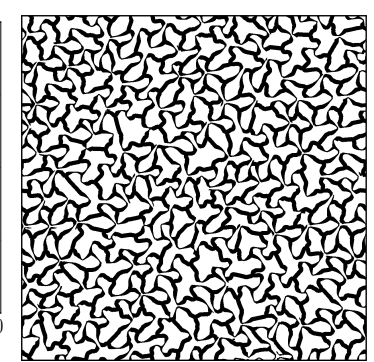

RSA

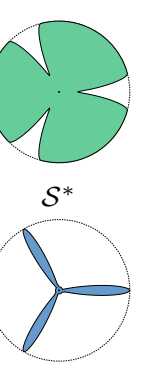

$\mathcal{S}$

Figure 11: Optimization with variable $\psi_{\mathcal{S} *} \in[4.0,15.0]$

\subsection{Experimental results}

We laser-cut different sheets made of Styrene-Butadiene Rubber (SBR) with shore hardness A70. The dimensions of the rubber sheets are $200 \times 200 \times 1$ millimeters. We extract the laser trajectories from the porous material images by contouring the interface between the solid and void phases and simplifying the contour polygons. The optimization considers a Poisson's ratio $v=0.48$ (SBR) of the solid phase, and minimum values $r_{\min }=0.1$ and $r_{\min }^{*}=10$ to avoid narrow features that are challenging to laser-cut. Due to the smaller space of optimization compared to Figures 10 to 12 the optimized numerical results have a higher but still negative Poisson's. The laser-cut sheets visually reveal an auxetic behavior Figure 13 . 


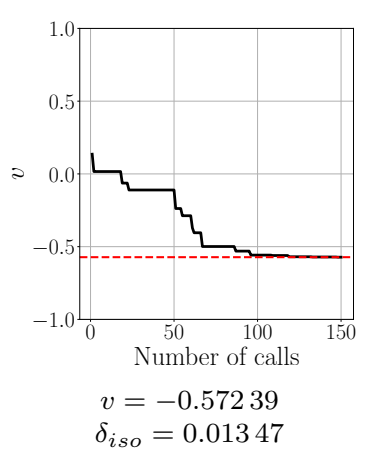

$\delta_{\text {iso }}=0.01347$

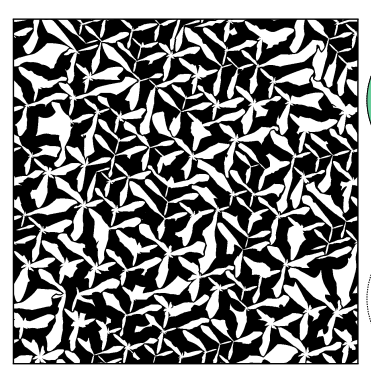

Poisson

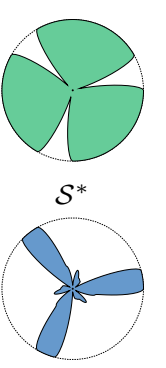

$\mathcal{S}$

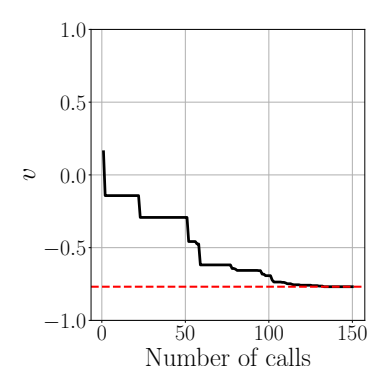

$v=-0.76846$

$\delta_{\text {iso }}=0.01437$

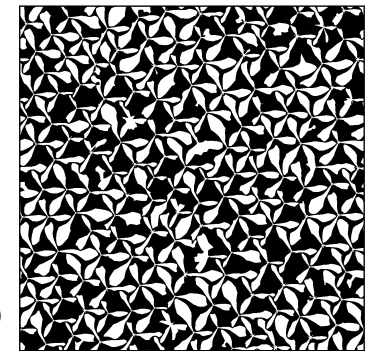

RSA

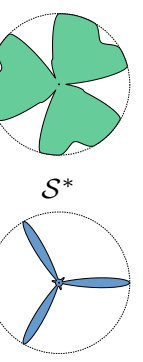

$\mathcal{S}$

Figure 12: Optimization with variable $\psi_{\mathcal{S}^{*}} \in[4.0,40.0]$

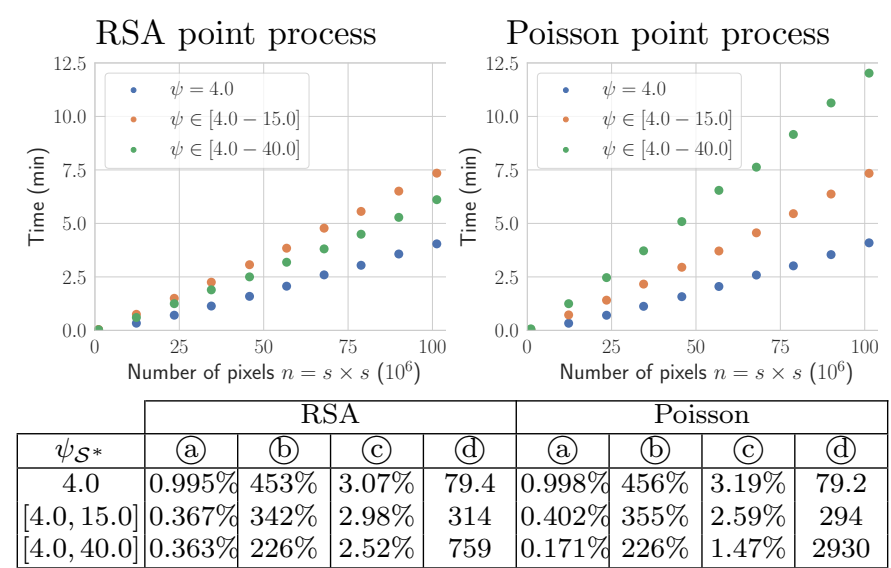

Table 1: Timing tests were performed on images with sizes ranging $n=[1,100]$ million pixels. We compute the growth process with a laptop equipped with an Intel i7-8650U. For each optimization result in Figures 10 to 12 we perform 10 tests with increasing size $n$ and plot the corresponding time in minutes. The table below shows the following additional statistics (considering the average value between all the tests). (a): Relative time spent computing the regularization with respect to the total time of computation. (b): Number of iterations in the main loop (line 6 of Algorithm 1 divided by $n$. (C): Average size of the queue $Q$ divided by $n$. (d): Average number of iterations in line 11 of Algorithm 1

\section{Conclusions and future work}

We have presented a computational approach based on parametric growth processes to minimize the Poisson's ratio of random porous materials. The results attain a negative Poisson's ratio while providing a numerical analysis showing close to linear elastic isotropic behavior. Increasing the number of parameters of $\psi_{\mathcal{S}}$ and $\psi_{\mathcal{S}^{*}}$ and their domain further improves the minimization. In practice, our approach can compute large-scale porous materials in a reasonable time. This capability is most valuable for applications that need to generate large quantities of random porous materials.

It is possible to generate heterogeneous materials with a parametric growth process by considering that both functions $\psi_{\mathcal{S}}$ and $\psi_{\mathcal{S}}{ }^{*}$ vary in the space. One of our approach's significant advantages over periodic structures is that the transitions between different material properties do not need to be handled [16, 22, as they are intrinsically cap- tured by the parameters governing the growth process. The gradations can be implicitly done either by constant (see Figure 15) or linear interpolation (see Figure 16) of the star-shaped sets. Figure 17 shows a 3D rendering of thin sheets obtained by our contouring method (see Section 4.4).

Manufacturing processes impose morphological constraints. Having a minimum local size of the solid phase is a common one. For a varying function $\psi_{\mathcal{S}^{*}}$, we may obtain porous materials whose solid phase has features with minimal local size (i.e., dimension of one-pixel, see Figure 14). Thus, it would be helpful to study how to ensure a minimum local size of the solid phase everywhere, either as a postprocessing step or as part of the growth process.

Our approach also opens up different opportunities for future work. First, to parallelize Algorithm 1 and significantly increase its performance. Second, to explore the much wider space of porous materials given by $\mathcal{S}$ and $\mathcal{S}^{*}$ having other symmetries and other point distributions than those considered in this article. Third, to extend Algorithm 1 to the $3 \mathrm{D}$ case by considering a $3 \mathrm{D}$ growth process and adapting the parameterization of $\mathcal{S}$ and $\mathcal{S}^{*}$. The inverse design of random $3 \mathrm{D}$ porous materials (e.g., random foams) is of particular interest for acoustic absorption 20] or tissue engineering [31]. Finally, it would be interesting to perform further physical tests of the results, considering larger deformations of the porous material (non-linear regime) and mechanical properties such as stress failure or buckling.

Our approach may also open the door to other applications besides minimizing the Poisson's ratio. For example, to tackle the inverse design and optimization of porous materials [50, 28, using our growth process. We conjecture that it will be sufficient to replace the objective function of Equation 11, with another one of interest in most cases.

\section{Code and data availability}

An open-source implementation of our approach can be found at ${ }^{1}$ We provide a script to automate the generation of all paper results and figures, apart from Figure 3

$1 \longdiv { \text { https://github.com/mfx-inria/auxeticgrowthprocess2d } }$ 

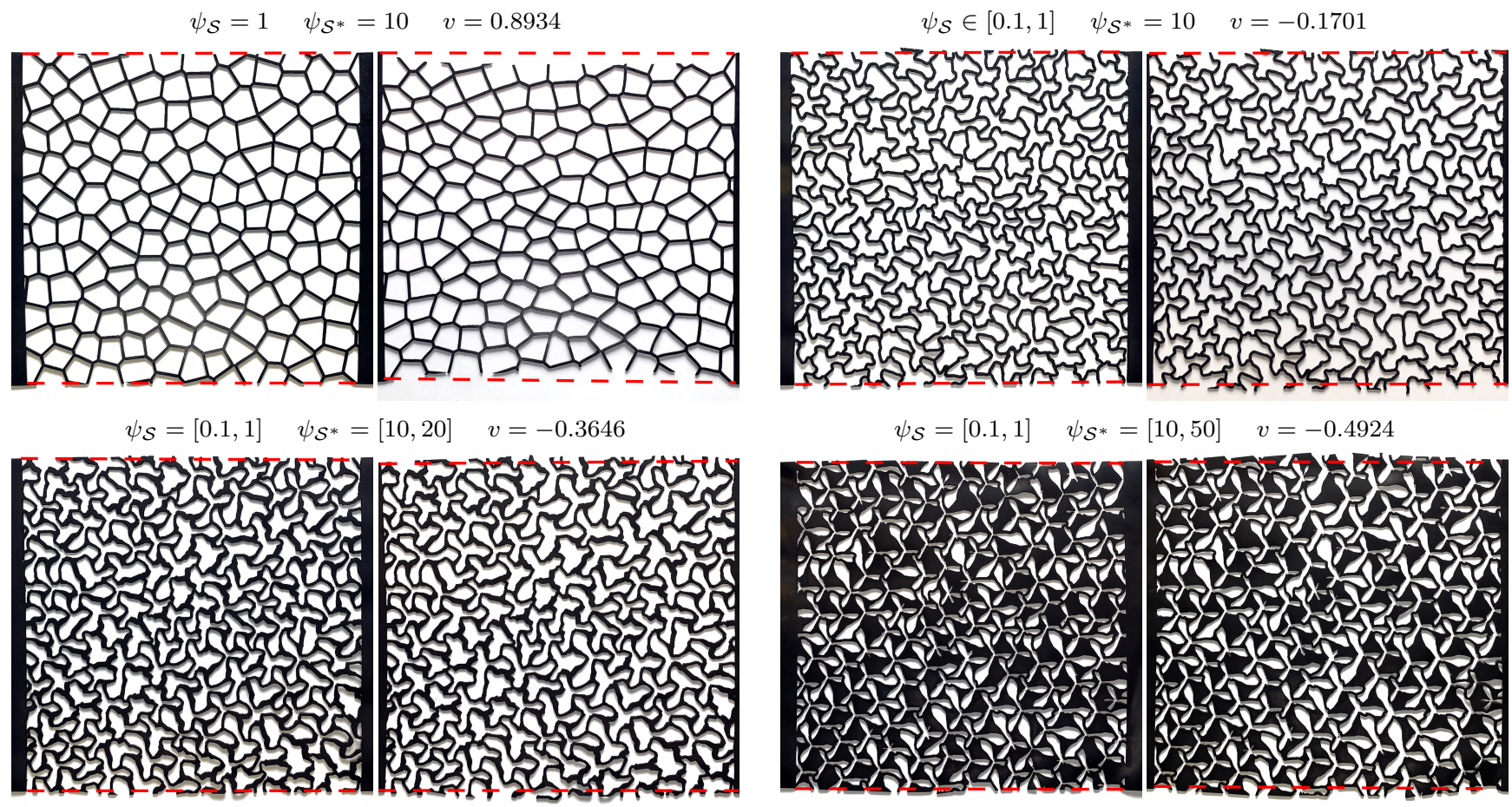

Figure 13: Visualization of the Poisson's ratio of four laser-cut rubber sheets. We show the picture in rest (left) and stretched position (right) for each sheet. The red dotted line is superimposed on the image to appreciate the lateral expansion better. Since our numerical results consider linear elasticity, we apply a relatively small uniform stretching of $10 \mathrm{~mm}$ (stretch ratio $5 \%$ ) to all the sheets. The Poisson's ratio $v$ corresponds to the mean numerical value obtained with stochastic homogenization. We visually observe a monotonic correlation between the lateral displacement and the corresponding numerical value of $v$.
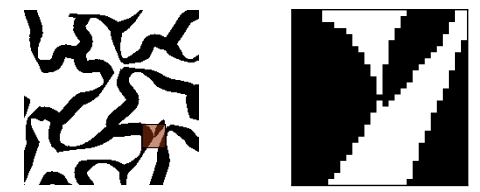

Figure 14: The solid phase in black color has a little feature inside the red square (left) that is visually magnified (right)

and Figures 15 to 17 All the results can be downloaded from 2

\section{Acknowledgments}

This work was partly supported by ANR MuFFin (ANR17-CE10-0002). Thanks to Pierre Bedell and Pierre-Alexandre Hugron for their help in doing the physical experimental tests.

\section{References}

\section{References}

[1] Kim Alderson, Andrew Alderson, Naveen Ravirala, Virginia Simkins, and Philip Davies. Manufacture and characterisation of thin flat and curved auxetic foam sheets. physica status solidi (b), 249(7):1315-1321, 2012

\footnotetext{
2 https://github.com/mfx-inria/auxeticgrowthprocess2d/raw/master/data.tar.gz
}

[2] Giacomo Aletti, Enea G Bongiorno, and Vincenzo Capasso. Statistical aspects of fuzzy monotone set-valued stochastic processes. application to birth-and-growth processes. Fuzzy Sets and Systems, 160(21):3140-3151, 2009.

[3] Giacomo Aletti, Enea G Bongiorno, and Vincenzo Capasso. Integration in a dynamical stochastic geometric framework. ESAIM: Probability and Statistics, 15:402-416, 2011.

[4] Grégoire Allaire. Homogenization and two-scale convergence. SIAM Journal on Mathematical Analysis, 23(6):1482-1518, 1992.

[5] Erik Andreassen and Casper Schousboe Andreasen. How to determine composite material properties using numerical homogenization. Computational Materials Science, 83:488-495, 2014

[6] Xavier Blanc, Claude Le Bris, and Frédéric Legoll. Some variance reduction methods for numerical stochastic homogenization. Philos. T. R. Soc. A, 374(2066):20150168, 2016.

[7] Alain Bourgeat and Andrey Piatnitski. Approximations of effective coefficients in stochastic homogenization. Annales de l'IHP Probabilités et statistiques, 40(2):153-165, 2004.

[8] BD Caddock and KE Evans. Microporous materials with negative Poisson's ratios. I. Microstructure and mechanical properties. Journal of Physics D: Applied Physics, 22(12):1877, 1989.

[9] N Chan and KE Evans. Fabrication methods for auxetic foams. Journal of materials Science, 32(22):5945-5953, 1997.

[10] Da Chen, Sritawat Kitipornchai, and Jie Yang. Dynamic response and energy absorption of functionally graded porous structures. Materials \& Design, 140:473-487, 2018.

[11] Sung Nok Chiu, Dietrich Stoyan, Wilfrid S Kendall, and Joseph Mecke. Stochastic geometry and its applications. John Wiley \& Sons, 2013.

[12] John Dagdelen, Joseph Montoya, Maarten de Jong, and Kristin Persson. Computational prediction of new auxetic materials. Nature communications, 8(1):1-8, 2017. 

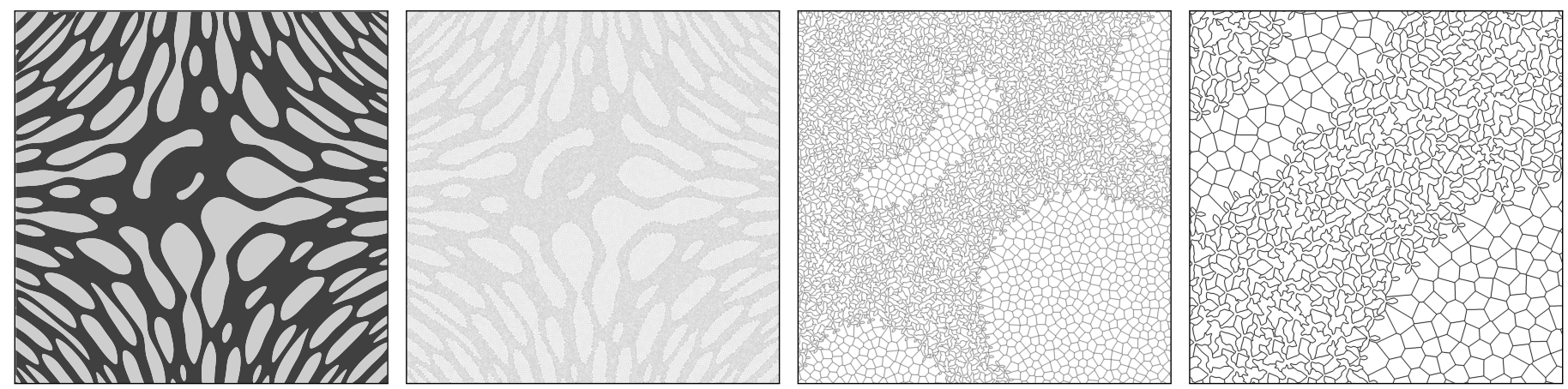

Figure 15: Spatially varying growth process with two different growth functions. Left: Input heterogeneous field. The darker regions correspond to the optimized $\psi_{\mathcal{S}}$ in Figure 10 , while the rest correspond to growth according to the Euclidean disk.
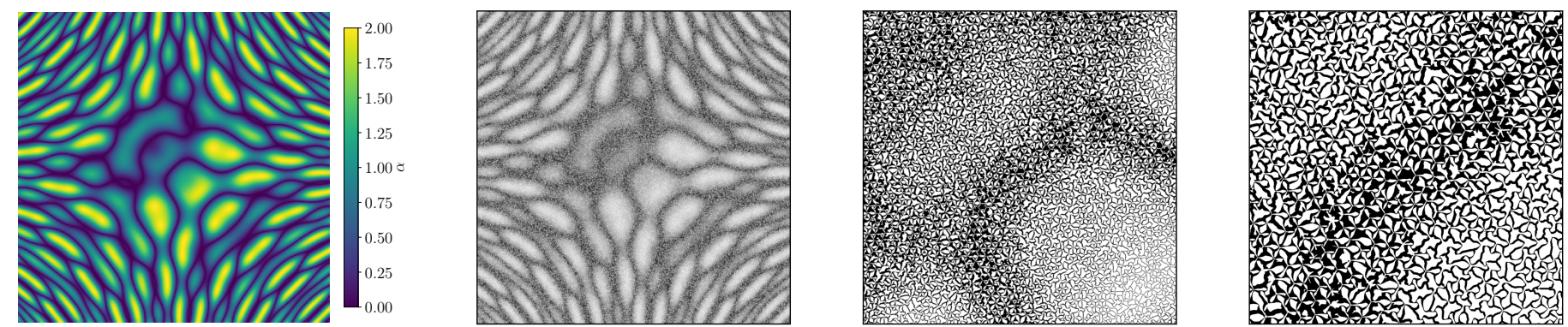

Figure 16: Spatially varying growth process with three different growth functions linearly interpolated. Left: Input field $\alpha \in[0,2]$ to control the linear interpolation between the optimized sets from Figure $10\left(\mathcal{S}_{\alpha=2}\right)$, Figure $11\left(\mathcal{S}_{\alpha=1}\right)$, and Figure $12\left(\mathcal{S}_{\alpha=0}\right)$. More precisely, for $\alpha \in[0,1)$ we consider the interpolated set $\alpha\left(\mathcal{S}_{\alpha=1}\right)+(1-\alpha)\left(\mathcal{S}_{\alpha=2}\right)$, and for $\alpha \in[1,2]$ we consider $(\alpha-1)\left(\mathcal{S}_{\alpha=0}\right)+(2-\alpha)\left(\mathcal{S}_{\alpha=1}\right)$.
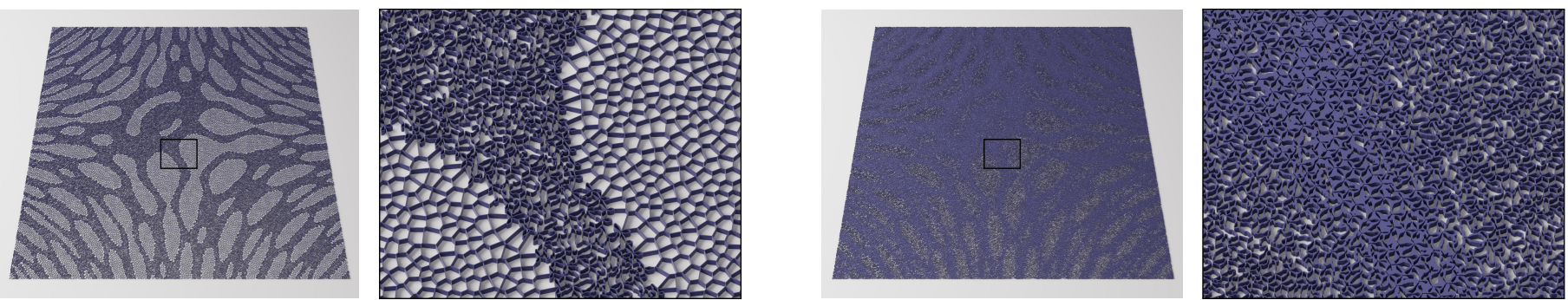

Figure 17: 3D rendering as thin sheet plates of the results of Figure 15 (left) and Figure 16 (right).

[13] S. Domaschke, A. Morel, G. Fortunato, and AE. Ehret. Random auxetics from buckling fibre networks. Nature communications, 10(1):1-8, 2019

[14] Sandra Forte and Maurizio Vianello. Symmetry classes for elasticity tensors. Journal of Elasticity, 43(2):81-108, 1996.

[15] Ruicong Gao, Dong Li, Liang Dong, and Xin Wang. Numerical analysis of the mechanical properties of 3D random Voronoi structures with negative Poisson's ratio. physica status solidi (b), page 1800539, 2019.

[16] Eric Garner, Helena MA Kolken, Charlie CL Wang, Amir A Zadpoor, and Jun Wu. Compatibility in microstructural optimization for additive manufacturing. Additive Manufacturing, 26:65-75, 2019

[17] Lorna J Gibson and Michael F Ashby. Cellular solids: structure and properties. Cambridge university press, 1999

[18] Joseph N Grima, Luke Mizzi, Keith M Azzopardi, and Ruben Gatt. Auxetic perforated mechanical metamaterials with randomly oriented cuts. Advanced materials, 28(2):385-389, 2016.

[19] Varda F Hagh and MF Thorpe. Disordered auxetic networks with no reentrant polygons. Physical Review B, 98(10):100101, 2018 .

[20] Fusheng Han, Gary Seiffert, Yuyuan Zhao, and Barry Gibbs. Acoustic absorption behaviour of an open-celled aluminium foam.
Journal of Physics D: Applied Physics, 36(3):294, 2003.

21] Umar Hossain, Shaaz Ghouse, Kenneth Nai, and Jonathan RT Jeffers. Controlling and testing anisotropy in additively manufactured stochastic structures. Additive Manufacturing, page 101849, 2021.

[22] Jingqiao Hu, Ming Li, Xingtong Yang, and Shuming Gao. Cellular structure design based on free material optimization under connectivity control. Computer-Aided Design, page 102854, 2020.

[23] Y Ibrahim, CM Davies, C Maharaj, Z Li, JP Dear, and PA Hooper. Post-yield performance of additive manufactured cellular lattice structures. Progress in Additive Manufacturing, pages $1-10,2020$.

[24] Toufik Kanit, Samuel Forest, Isabelle Galliet, Valérie Mounoury, and Dominique Jeulin. Determination of the size of the representative volume element for random composites: statistical and numerical approach. Int. J. Solids Struct., 40(13-14):3647$3679,2003$.

[25] H MA Kolken and AA Zadpoor. Auxetic mechanical metamaterials. RSC Advances, 7(9):5111-5129, 2017.

[26] AG Kolpakov. Determination of the average characteristics of elastic frameworks. J. Appl. Math. Mech., 49(6):739-745, 1985 
[27] Jagdish A Krishnaswamy, Federico C Buroni, Roderick Melnik, Luis Rodriguez-Tembleque, and Andres Saez. Design of polymeric auxetic matrices for improved mechanical coupling in lead-free piezocomposites. Smart Materials and Structures, 29(5):054002, 2020.

[28] Siddhant Kumar, Stephanie Tan, Li Zheng, and Dennis M Kochmann. Inverse-designed spinodoid metamaterials. $n p j$ Computational Materials, 6(1):1-10, 2020.

[29] Roderic Lakes. Foam structures with a negative Poisson's ratio. Science, 235:1038-1041, 1987.

[30] Roderic S Lakes. Negative-Poisson's-ratio materials: auxetic solids. Annual review of materials research, 47:63-81, 2017.

[31] Robert Lanza, Robert Langer, Joseph P Vacanti, and Anthony Atala. Principles of tissue engineering. Academic press, 2020.

[32] Dong Li, Liang Dong, Jianhua Yin, and Roderic S Lakes. Negative Poisson's ratio in 2D Voronoi cellular solids by biaxial compression: a numerical study. Journal of materials science, 51(14):7029-7037, 2016.

[33] Jun Liu, Yunhuan Nie, Hua Tong, and Ning Xu. Realizing negative Poisson's ratio in spring networks with close-packed lattice geometries. Physical Review Materials, 3:055607, 2019.

[34] Jonàs Martínez, Jérémie Dumas, and Sylvain Lefebvre. Procedural Voronoi foams for additive manufacturing. ACM Transactions on graphics, 35(4):44:1-44:12, 2016.

[35] Jonàs Martínez, Mélina Skouras, Christian Schumacher, Samuel Hornus, Sylvain Lefebvre, and Bernhard Thomaszewski. Starshaped metrics for mechanical metamaterial design. ACM Transactions on Graphics, 38(4):1-13, 2019.

[36] MJ Mirzaali, H Pahlavani, and AA Zadpoor. Auxeticity and stiffness of random networks: Lessons for the rational design of 3D printed mechanical metamaterials. Applied Physics Letters, 115(2):021901, 2019

[37] Maher Moakher and Andrew N Norris. The closest elastic tensor of arbitrary symmetry to an elasticity tensor of lower symmetry. Journal of Elasticity, 85(3):215-263, 2006

[38] Jonas Močkus. On Bayesian methods for seeking the extremum. In Optimization techniques IFIP technical conference, pages 400-404. Springer, 1975.

[39] Jochen Mueller, Kathryn H Matlack, Kristina Shea, and Chiara Daraio. Energy absorption properties of periodic and stochastic 3D lattice materials. Advanced Theory and Simulations, 2(10):1900081, 2019

[40] Lewis Mullen, Robin C Stamp, Peter Fox, Eric Jones, Chau Ngo, and Christopher J Sutcliffe. Selective laser melting: A unit cell approach for the manufacture of porous, titanium, bone in-growth constructs, suitable for orthopedic applications. ii. randomized structures. Journal of Biomedical Materials Research Part B: Applied Biomaterials: An Official Journal of The Society for Biomaterials, The Japanese Society for Biomaterials, and The Australian Society for Biomaterials and the Korean Society for Biomaterials, 92(1):178-188, 2010.

[41] Andrew Norris. The isotropic material closest to a given anisotropic material. Journal of Mechanics of Materials and Structures, 1(2):223-238, 2006.

[42] Atsuyuki Okabe, Barry Boots, Kokichi Sugihara, and Sung Nok Chiu. Spatial tessellations: concepts and applications of Voronoi diagrams. John Wiley \& Sons, 2000.

[43] Nidhi Pashine, Daniel Hexner, Andrea J Liu, and Sidney R Nagel. Directed aging, memory, and nature's greed. Science advances, 5(12), 2019.

[44] Carlos M Portela, A Vidyasagar, Sebastian Krödel, Tamara Weissenbach, Daryl W Yee, Julia R Greer, and Dennis M Kochmann. Extreme mechanical resilience of self-assembled nanolabyrinthine materials. Proceedings of the National Academy of Sciences, 117(11):5686-5693, 2020.

[45] Guangzhao Qin and Zhenzhen Qin. Negative Poisson's ratio in two-dimensional honeycomb structures. npj Computational Materials, 6(1):1-6, 2020.

[46] Daniel Rayneau-Kirkhope, Silvia Bonfanti, and Stefano Zapperi. Density scaling in the mechanics of a disordered mechanical meta-material. Applied Physics Letters, 114(11):111902, 2019.
[47] Daniel R Reid, Nidhi Pashine, Alec S Bowen, Sidney R Nagel, and Juan J de Pablo. Ideal isotropic auxetic networks from random networks. Soft Matter, 15(40):8084-8091, 2019.

[48] Daniel R Reid, Nidhi Pashine, Justin M Wozniak, Heinrich M Jaeger, Andrea J Liu, Sidney R Nagel, and Juan J de Pablo. Auxetic metamaterials from disordered networks. Proceedings of the National Academy of Sciences, 115(7):1384-1390, 2018.

[49] Xin Ren, Raj Das, Phuong Tran, Tuan Duc Ngo, and Yi Min Xie. Auxetic metamaterials and structures: A review. Smart materials and structures, 27(2):023001, 2018.

[50] Henrik Ronellenfitsch, Norbert Stoop, Josephine Yu, Aden Forrow, and Jörn Dunkel. Inverse design of discrete mechanical metamaterials. Physical Review Materials, 3:095201, 2019.

[51] Krishna Kumar Saxena, Raj Das, and Emilio P Calius. Three decades of auxetics research - materials with negative Poisson's ratio: a review. Advanced Engineering Materials, 18(11):18471870, 2016.

[52] Craig Schroeder, William C Regli, Ali Shokoufandeh, and Wei Sun. Computer-aided design of porous artifacts. Computeraided design, 37(3):339-353, 2005.

[53] Dietrich Stoyan and Martin Schlather. Random sequential adsorption: relationship to dead leaves and characterization of variability. Journal of Statistical Physics, 100(5-6):969-979, 2000

[54] M Stroeven, H Askes, and LJ Sluys. Numerical determination of representative volumes for granular materials. Computer Methods in Applied Mechanics and Engineering, 193(3032):3221-3238, 2004.

[55] MF Thorpe and IWONA JaSIUK. New results in the theory of elasticity for two-dimensional composites. Proceedings of the Royal Society of London. Series A: Mathematical and Physical Sciences, 438(1904):531-544, 1992.

56] Head Tim, Louppe Gilles, and Shcherbatyi Iaroslav. scikitoptimize 0.7.4.

57] VP Tran, J Guilleminot, Sébastien Brisard, and Karam Sab. Stochastic modeling of mesoscopic elasticity random field. $\mathrm{Me}$ chanics of Materials, 93:1-12, 2016.

[58] Panagiotis Vogiatzis, Shikui Chen, Xiao Wang, Tiantian Li, and Lifeng Wang. Topology optimization of multi-material negative poisson's ratio metamaterials using a reconciled level set method. Computer-Aided Design, 83:15-32, 2017.

[59] Woldemar Voigt. Lehrbuch der kristallphysik, volume 962. Teubner Leipzig, 1928.

60] B Widom. Random sequential addition of hard spheres to a volume. The Journal of Chemical Physics, 44(10):3888-3894, 1966

[61] Wei Yang, Zhong-Ming Li, Wei Shi, Bang-Hu Xie, and MingBo Yang. Review on auxetic materials. Journal of materials science, 39(10):3269-3279, 2004.

[62] O Zerhouni, MG Tarantino, and K Danas. Numerically-aided $3 \mathrm{D}$ printed random isotropic porous materials approaching the Hashin-Shtrikman bounds. Composites Part B: Engineering, 156:344-354, 2019

\section{Appendix A. Derivation of $c_{11}^{i s o}$ and $c_{12}^{i s o}$}

For the three-dimensional case, the minimizing values of $c_{11}^{i s o}, c_{12}^{i s o}$ were derived, for instance, in 41. We were not able to find any previous work concerning the more straightforward two-dimensional case. Nevertheless, the values minimizing Equation 8 can be derived as follows. Consider the function $f$ with two arguments $c_{11}^{i s o}$ and $c_{12}^{i s o}$

$$
\begin{aligned}
f= & \left\|C-C^{i s o}\right\|_{F}^{2} \\
= & \left(c_{11}-c_{11}^{i s o}\right)^{2}+2\left(c_{12}-c_{12}^{i s o}\right)^{2} \\
& +\left(c_{22}-c_{11}^{i s o}\right)^{2}+2 c_{13}^{2}+2 c_{23}^{2}+\left(c_{33}-\frac{c_{11}^{i s o}-c_{12}^{i s o}}{2}\right)^{2}
\end{aligned}
$$


whose first-order partial derivatives are

$$
\begin{array}{r}
\frac{\partial f}{\partial c_{11}^{i s o}}=-c_{33}-2\left(c_{22}-c_{11}^{i s o}\right)+\frac{c_{11}^{i s o}-c_{12}^{i s o}}{2}-2\left(c_{11}-c_{11}^{i s o}\right) \\
\frac{\partial f}{\partial c_{12}^{i s o}}=c_{33}-4\left(c_{12}-c_{12}^{i s o}\right)-\frac{c_{11}^{i s o}-c_{12}^{i s o}}{2}
\end{array}
$$

The Hessian matrix $H$ of $f$

$$
H=\left[\begin{array}{cc}
\frac{\partial^{2} f}{\partial\left(c_{11}^{i s o}\right)^{2}} & \frac{\partial^{2} f}{\partial c_{11}^{i s o} c_{12}^{i s o}} \\
\frac{\partial^{2} f}{\partial c_{12}^{i s o} c_{11}^{i s o}} & \frac{\partial^{2} f}{\partial\left(c_{12}^{i s o}\right)^{2}}
\end{array}\right]=\left[\begin{array}{cc}
\frac{9}{2} & -\frac{1}{2} \\
-\frac{1}{2} & \frac{9}{2}
\end{array}\right]
$$

is positive definite everywhere, and it follows that $f$ is a convex function. Since $\frac{\partial^{2} f}{\partial\left(c_{11}^{i s o}\right)^{2}}=\frac{\partial^{2} f}{\partial\left(c_{12}^{i s o}\right)^{2}}>0$ the function $f$ has a global minimum at

$$
\begin{gathered}
c_{11}^{i s o}=\frac{1}{20}\left(9\left(c_{11}+c_{22}\right)+2 c_{12}+4 c_{33}\right) \\
c_{12}^{i s o}=\frac{1}{20}\left(c_{11}+c_{22}+18 c_{12}-4 c_{33}\right)
\end{gathered}
$$

found by solving the system of linear equations from Equation A.2 being equal to zero. 